Editorial

\title{
Multi-disciplinary behavioral health evaluations
}

\author{
Volume I Issue 5 - 2014 \\ Kathryn Seifert \\ CARE2, LLC, England
}

\section{Editorial}

The face of behavioral healthcare is changing. In particular integration of disciplines is increasingly expected. Psychological evaluations could also evolve into interdisciplinary evaluations which include specialists for certain highly complex cases were danger to self or others is a concern. More complex and difficult cases appear to fit Moffitt's' theory of life-course anti-social behavior. For this reason, these cases may need a different type of evaluation.

For people with complex and severely chronic behaviors that harm self or others, information on the family, community, and school or job is needed as much as the internal psychological factors, such as personality, cognitive abilities, and coping style. The reasoning behind this is the fact that beyond the risk factors of harm to self or others is the day to day balance between an individual's stressors and coping. Such evaluations are needed in the case of custody and placement issues, child abuse or neglect, family conflict or chaos, violence risk reduction planning, and multiple family generations of trauma.

It is well established that for highly complex and severely chronic cases, there are often problems in functioning in multiple domains of the person's life. Therefore, assessment and interventions must cover multiple aspects of the person's life and functioning to reduce problems and build strengths.

A person's family can be a major source of strength and stress. The effectiveness of the parent's or partner's functioning can help shape the level of functioning of the person being evaluated. Therefore, a thorough psycho-social history is needed on every family member. Topics to be covered include trauma, violence, school and job performance, child abuse, mental health history and functioning, addictions history for multiple generations, legal history, violence, maladaptive sexual behavior, medications and past treatment. Prior treatment records should be obtained through signed releases of information. Social workers are well trained to do thorough biopsychosocials.

If there is a history or suspicion of addictions anywhere in the family, an addictions specialist may be needed, as well. When there are addictions in the family, it raises the risk that the youth will have problems with substance abuse. A substance abuse assessment can be done by the same social worker that does the psychosocial histories, but he/she should be trained in addictions assessment and interventions and use appropriate tools such as the SUDS for determining ASAM criteria.

A psychologist and a psychiatrist should perform exams and testing as needed, especially if a major mental illness or cognitive impairment is suspected in any family member. Psychological tests might include the MMPI/MMPI-A, MCMI/MACI, Bender, or WAIS/WISC. Additionally, a family specialist should assess family dynamics. These functions can be performed by the same or different professionals. The key is to have professionals that are trained in the appropriate discipline.

Assessment of risk of crime/delinquency, violence or sexual offending and risk reduction plans should be part of the evaluation if these behaviors are in question for any family member. This requires training and assessments that are specific to these problem areas.

There are 2 assessments that are directly related to risk of youth violence and have sufficient statistical ROC's (.75-.80): SAVRY ${ }^{2}$ and CARE2. ${ }^{3}$ An assessment for measuring psychopathy in youth (a controversial topic) is the PCL-YV. ${ }^{4}$ A very good measure for general delinquency is the LS/CMI. ${ }^{5}$ The ERASOR ${ }^{6}$ is a good instrument for risk for youthful sexual offending. Most of these instruments use guided clinical judgment which gives the clinician the known risk factors for a behavior and leaves the assigning of risk level to the experienced clinician. Remembering that risk prediction through unaided clinical judgment is at chance levels, guided clinical judgment is ultimately better than unaided clinical judgment. An assessment for risk of adult violence is the HCR-20. ${ }^{7}$ Of course training in the field should also be expected for any evaluator of this domain.

This type of evaluation allows the team to move in the direction of risk reduction planning taking risk factors, strengths and stressors of the entire family into account. In that way, rather than assigning a risk level to an individual, which at best is $75 \%$ accurate, this type of evaluation gives the clinician the ability make recommendations for interventions to reduce risk factors and stressors and increase strengths and coping within the individual and his/her environment.

Another emerging assessment field is the measurement of developmental level of skills. The negative effect of trauma on the development of skills has been described by Bessel van der Kolk. ${ }^{8}$ To address those developmental deficits they must be measured. An instrument that effectively does that is the Behavioral Objective Sequence. ${ }^{9}$ The instrument also gives guidance on interventions that are developmentally appropriate. It also allows skills to be built on each prior skill.

A medical evaluation is also important and copies of medical records should be obtained on the youth and all family members. Some may say that this is too invasive. In fact it is much more invasive that what is traditionally done and should only be done for 
the most complex cases with past, ongoing, or future risk of harm to self or others. The reality is that this level of data is often impossible to get, so the clinician gathers what is realistic and possible to collect. The certainty with which the clinician can state his/her conclusions or recommendations increases with the amount and type of data collected.

Following all interviews and data gathering, the evaluation team meets and discusses the case, findings and recommendations. Each discipline is responsible for writing his/her pertinent section and sharing it with other members of the team. The most appropriate team member writes the summary. All disciplines submit points for the treatment plan. Feedback is shared with family members by team member(s) to include recommendations for treatment. A report is then submitted to the appropriate entity.

The impetus behind this approach is to make evaluations more holistic, take into consideration environmental strengths and stressors and create more well-rounded treatment plans. An additional value of this approach is to look at an evaluation from the eyes of multiple specialists.

\section{Acknowledgments}

None.

\section{Conflicts of interest}

Author declares there are no conflicts of interest.

\section{Funding}

None.

\section{References}

1. Moffitt TE. Adolescent Limited and Life Course Persistent anti-social Behavior: A developmental taxonomy. In Piquero AR, Mazarolle P (Ed.), Life-course criminology: Contemporary and classic readings. Belmont, CA: Wadsworth. 2001.

2. Borum RB. SAVRY. 2000.

3. Seifert K. CARE-2: Child and Adolescent Risk/Needs Evaluation. Salisbury: CARE2. 2012.

4. Forth A. Hare Psychopathy Checklist - Youth Version. Ontario, CA: MHS. 2004.

5. Andrews DB. LS/CMI. Toronto, CA: MHS. 2004.

6. Worling JR. Estimate of Risk of Adolescent Sexual Offense Recidivism (Version 2.0: The "ERASOR"). In: MC Calder (Ed.), Juveniles and children who sexually abuse: Frameworks for assessment. Lyme Regis, Dorset, UK: Russell House Publishing. 2001.

7. Douglas KS. HCR-20V3: Assessing risk of violence - User guide. Burnaby, Canada: Mental Health, Law, and Policy Institute, Simon Fraser University. 2013.

8. Ford JD, Grasso D, Greene C, et al. Clinical Significance of a proposed developmental trauma disorder diagnosis: results of an international survey of clinicians. J Clin Psychiatry. 2013;74(8):841-849.

9. Braaten S. Behavioral objective sequence. Champaigne, IL: Research Press. 1998. 